\title{
Performance Comparison between ETSI SmartBAN and Bluetooth
}

\author{
Andrea D'Souza ${ }^{1}$, Harri Viittala ${ }^{2}$, Matti Hämäläinen ${ }^{2}$, Lorenzo Mucchi $^{1}$ \\ ${ }^{1}$ Department of Information Engineering, University of \\ Florence, Florence, Italy \\ matrea3@gmail.com, lorenzo.mucchi@unifi.it \\ ${ }^{2}$ Centre for Wireless Communications \\ University of Oulu, Oulu, Finland \\ harri.viittala@gmail.com, matti.hamalainen@oulu.fi
}

\begin{abstract}
This paper introduces the comparative performance analysis between ETSI SmartBAN and Bluetooth low energy (BLE) in the interfered additive white Gaussian noise (AWGN) channel as well as in multipath fading channel. Both technologies are possible solutions for wireless body area networks (WBAN) to implement services like delivering the vital signs data of an individual. The results show how the SmartBAN can outperform BLE in both AWGN and fading channel. In addition, SmartBAN can take advantages of repetition and coding features, which are not present in BLE to increase its performance even more.
\end{abstract}

Keywords-bluetooth low energy, interference; multipath; bit error rate; frame error rate.

\section{INTRODUCTION}

The use of wireless body area network (WBAN) is one way to convey further a vital sign information measured from a human body. This procedure is a modern way towards personalized healthcare and remote health monitoring. If a patient can be remotely monitored and the health-related information is possible to ubiquitously access by healthcare professionals, patients can be, e.g., discharged earlier from hospitals thus reducing medics workload and reduce costs.

In 2013, the European Telecommunications Standards Institute (ETSI) initiated a work towards smart body area networks under the technical committee (TC) SmartBAN. The focus of SmartBAN is to develop a low-power technology to be used in wireless devices for wearable and implantable devices. The SmartBAN utilization areas include health \& wellness, training, personalized medicine and safety applications. [1]

So far, TC SmartBAN has released three standards [2][4]. In addition, technical report describing the radio environment at hospital is released [5].

The objective of this paper is to compare the SmartBAN technology with another low power technology useable in wireless medical application, namely Bluetooth low energy (BLE) standard [12]. Both standards have low power consumption and are operating at the $2.4 \mathrm{GHz}$ ISM (Industrial, Science and Medical) band. BLE is one of the most used standards nowadays for wireless short-range communications.
The paper is organized as follows: Section II provides description of Bluetooth low energy and of the main characteristics of its physical layer. In Section III, simulation models of the SmartBAN and BLE used in the study are given. In Sec. IV, the simulation results are shown and discussed. The conclusions are given in Sec. V.

\section{OVERVIEW OF BLETOOTH LOW ENERGY STANDARD}

The concept of low energy in Bluetooth has been introduced in 2010 with the Bluetooth 4.0 standard but has been proved that the two technologies (Bluetooth and BLE) are not interchangeable. [14]

The BLE is developed to improve the Bluetooth performance in regards of energy consumption, to reduce the costs and for less complexity respect to the original Bluetooth technology [13]. BLE design is similar in respect to the typical Bluetooth (BT) with very few differences at physical layer (PHY) level. In this study, a BLE PHY model following the specification from [12] is developed. The model, with modulation and frequency hopping features, is verified with the standard Bluetooth PHY model designed by Mathworks [14].

The operating frequency band of the BLE is between $2402 \mathrm{MHz}$ and $2480 \mathrm{MHz}$. Instead of the 79 channels of 1 $\mathrm{MHz}$ in the BT, BLE is characterized by 40 channels with 2 MHz spacing. The 40 BLE channels are shown in Figure 1. [15] The three highlighted channels are named advertising channels used mainly for highlight own presence or discovering devices. Advertising channels are channels 37, 38 and 39, decentralized respectively in the following frequencies 2402, 2426 and $2480 \mathrm{MHz}$. The other 37 channels are used to transfer data between devices, and they are named data channels. [16]

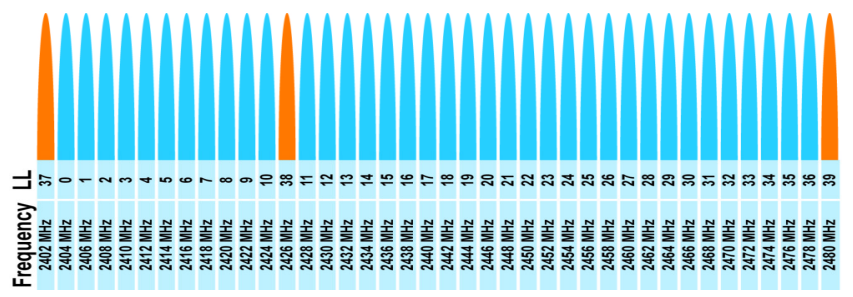

Figure 1. Bluetooth Low Energy Frequency Channels. 
One of the main points of the BLE and BT technologies is the use of frequency hopping mechanism. In BLE, an adaptive frequency hopping mechanism is used in data channels to prevent multipath fading or channels interference [17]. In the simulator, the frequency hopping code is implemented according to [12].

Another specification of the physical layer of BLE is the modulation, which is Gaussian Frequency Shift Keying (GFSK), so the data pulses are first filtered in a Gaussian filter and then after are FSK modulated with a modulation index in the range [0.45 - 0.55]. A transmission rate is 1 Mbps with 1 bit per symbol [12].

\section{SIMULATION MODEL}

In this section, the simulation models used in the study are presented.

\section{A. SmartBAN simulator}

ETSI SmartBAN PHY model is based on the overall system description presented in [6] and physical layer definitions from [2][7]. Radio channel model is based on the on-on-body channel model CM3 by the IEEE [8]. In addition, realistic, measurement based co-channel interference as introduced in [5][9] is used in the simulations. The performance of SmartBAN system in interfered fading channel is presented in [10] and [11], but the comparative analysis between SmartBAN and any other competing technology has not been published yet. This paper is fulfilling this gap by comparing SmartBAN and BLE biterror-rate (BER) performances in AWGN and fading channels. The SmartBAN simulator is based on the simulator presented in [19], and it is illustrated in Figure 2. The data bits $b[k]$ are encoded with the $\mathrm{BCH}(127,113)$ encoder followed by GFSK modulator with the modulation index $(h)$ of 0.5 and bandwidth-time product $(B T)$ of 0.5 . The PHY layer protocol data unit (PPDU) is repeated one, two or four times according to [2]. The received signal $r(t)$ is

$$
r(t)=s(t) * h(t)+i(t)+n(t),
$$

where * denotes the convolutional operator, $h(t)$ is the fading channel, $i(t)$ is the interference process and $n(t)$ is the additive white Gaussian noise (AWGN) with zero mean and variance $\sigma_{\mathrm{n}}$. Different interference models are introduced in [19]. These interference models are based on the measurement campaigns carrier out in the real hospital environment [5]. The received PPDUs are combined by using the equal gain combining (EGC) method. The demodulator applies a correlator followed by a maximumlikelihood sequence detector (MLSD).

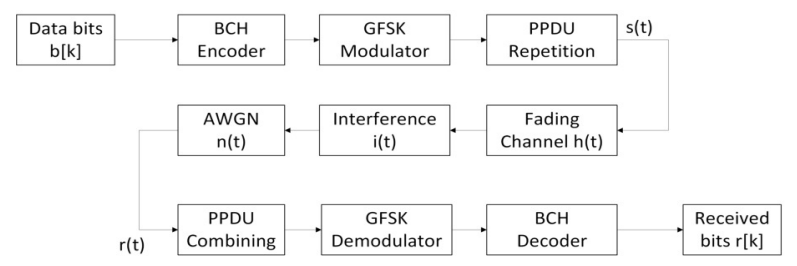

Figure 2. SmartBAN simulator.

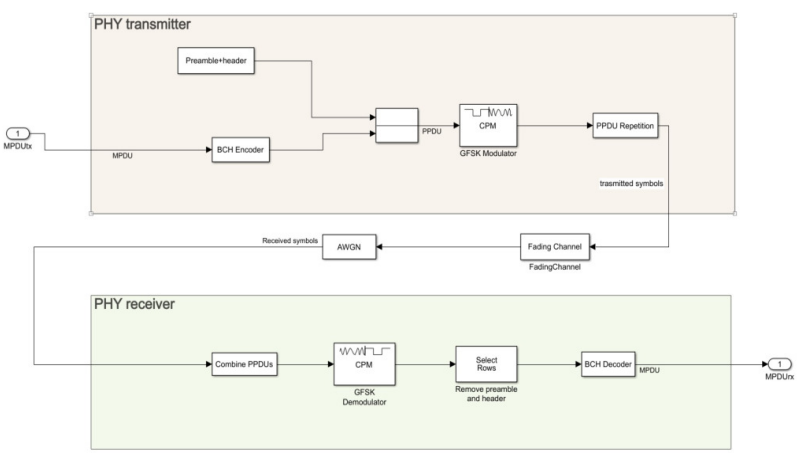

Figure 3. Physical layer of SmartBAN. Implementation in SmartBAN simulator.

For a fair comparison between the SmartBAN and BLE a single system simulator has been developed using Matlab® with Simulink ${ }^{\circledR}$. The PHY simulation models for SmartBAN and BLE are represented in Figure 3 and Figure 4 , respectively, in more details.

\section{B. BLE simulator}

The BLE PHY in Figure 4 on the transmitter part is designed to assemble packets of 62500 bits at a speed of 1 Mbps. Those packets are sent to a GFSK modulator implemented with a frequency hopping mechanism. The GFSK modulator uses modulation index of 0.5 , bandwidthtime product of $0.5,100$ samples per symbol and a pulse length of one bit per symbol.

A fundamental part of the PHY in BLE simulator is the frequency hopping mechanism, where the frequency is changed following a random pattern to reduce interference. The radio channel set is composed by 40 different channel realizations The frequency hopping code implemented is shown in Figure 5. A random data channel between 0 and 36 is selected so that no overlapping channel is present. The hop rate used in the simulator is $1600 \mathrm{~Hz}$.

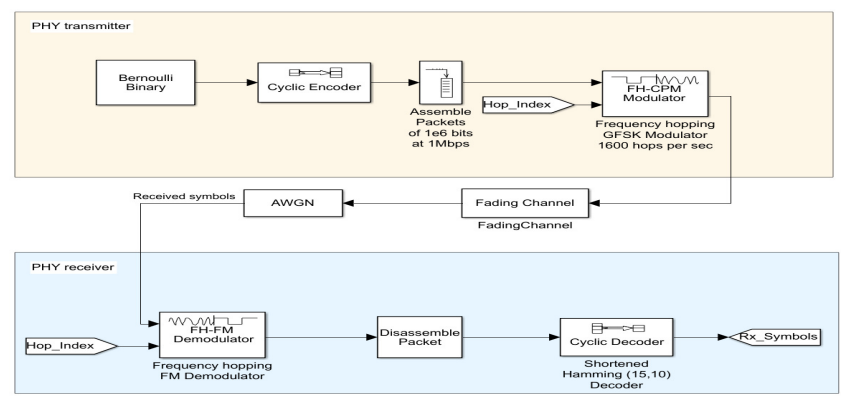

Figure 4. Physical layer of the Bluetooth low energy. Implementation in SmartBAN simulator. 


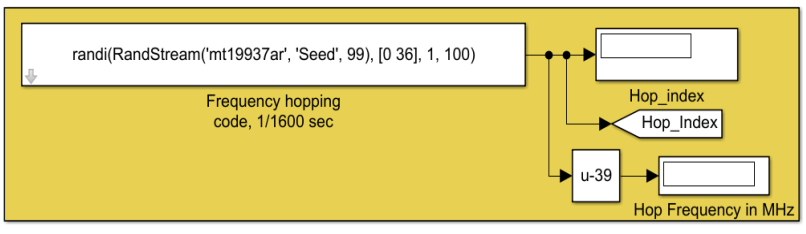

Frequency Hopping Code

Figure 5. Frequency hopping mechanism in BLE.

The modulated signal propagates through the fading channel and an additive white Gaussian noise (AWGN) block. The received signal is demodulated in a FH-FM demodulator characterized by a frequency separation of 10 $\mathrm{Hz}$ and 100 samples per symbol.

For validation of the designed BLE PHY, a performance comparison with the theoretical curve GFSK was done. The GFSK modulator parameters are set as $B T=0.5$ and $h=0.5$ for the comparison.

The results of the comparison are given in Figure 6. The performance of the BLE is slightly worse than the theoretical one, as expected. The gap between the BER curves is acceptable and the result validates the design of the BLE PHY that will be used for the comparison with SmartBAN in this paper.

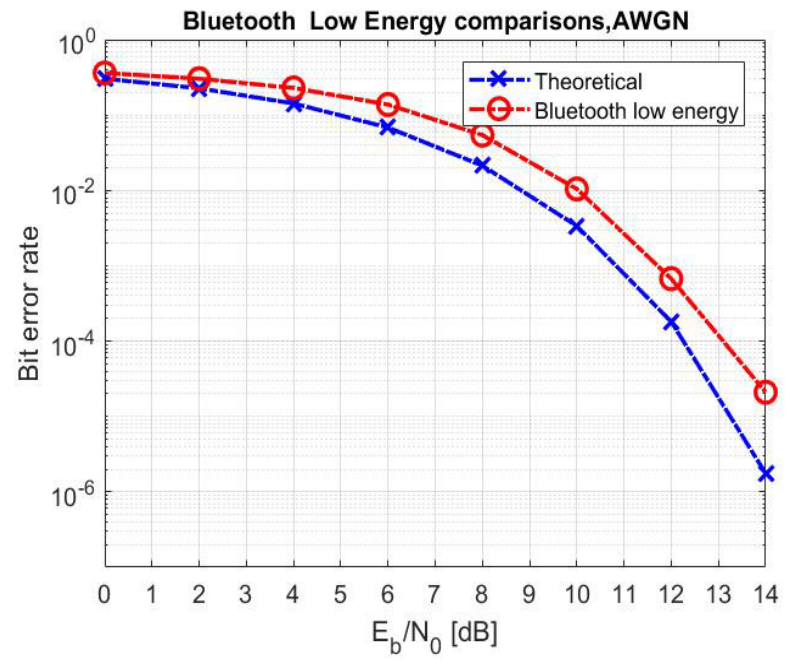

Figure 6. Comparison between BLE and the theoretical curve with GFSK with AWGN channel.

A summary of the main parameters of the PHYs are presented in Table 1. The main difference between SmartBAN and BLE is that the former implements PHY Protocol Data Unit (PPDU) repetition while the BLE does not.

In both cases, the modulated signals pass through a fading and a AWGN channel. The fading channel model used in the analysis is the IEEE 802.15 .6 body surface to body surface model CM3 (Scenario S4 \& S5) for $2.4 \mathrm{GHz}$ [18]. The measurement for modelling the fading channel were carried out in a hospital and mainly models a link between a coordinator situated in the middle of a human torso and different locations on the body. The channel model for flat small fading is described by a Ricean distribution with parameters described in [10]. In the simulations, the noise variance in AWGN channel is changed to define the required $E_{\mathrm{b}} / N_{0}$ at the receiver.

TABLE 1. SMARTBAN AND BLE $2.4 \mathrm{GHz}$ PHYS

\begin{tabular}{|c|c|c|}
\hline PARAMETER & SmartBAN & BLE \\
\hline $\begin{array}{c}\text { Operating frequency } \\
{[\mathrm{MHz}]}\end{array}$ & $2401-2481$ & $2402-2480$ \\
\hline $\begin{array}{c}\text { Channel bandwidth } \\
{[\mathrm{MHz}]}\end{array}$ & 2 & 2 \\
\hline Number of channels & 40 & 40 \\
\hline Repetition/spreading & $\begin{array}{c}2 \mathrm{x} \text { or } 4 \mathrm{x} \\
\text { Entire PPDU }\end{array}$ & No repetition \\
\hline Modulation & $\begin{array}{c}\text { GFSK } \\
(B T=0.5, h=0.5)\end{array}$ & $\begin{array}{c}\text { GFSK } \\
(B T=0.5, \\
h=0.5)\end{array}$ \\
\hline Symbol rate[Mbps] & 1 & 1 \\
\hline Receiver type & coherent & non-coherent \\
\hline
\end{tabular}

\section{SimUlation RESULTS}

\section{A. Simulation parameters}

The parameters used in the SmartBAN and BLE simulations are summarized in Table 2 and Table 3, respectively. More details about the SmartBAN simulator can be found from [10].

\section{TABLE 2. SMARTBAN SIMULATION PARAMETERS}

\begin{tabular}{|c|c|}
\hline Parameter & Value(s) \\
\hline PPDU repetition & 1 \\
\hline Retrasmission & no \\
\hline MAC frame body & $20,500,1000$ \\
\hline Samples for GFSK symbol & 1 \\
\hline Pulse length of GFSK & 10 \\
\hline $\begin{array}{c}\text { Traceback depth of GFSK } \\
\text { demodulator }\end{array}$ & 45 \\
\hline Distance $[\mathrm{cm}]$ & \\
\hline
\end{tabular}

TABLE 3. BLE SIMULATION PARAMETERS

\begin{tabular}{|c|c|}
\hline Parameter & Value(s) \\
\hline Repetition & Not present \\
\hline Retransmission & Not available \\
\hline Sample for GFSK symbol & 100 \\
\hline Pulse length of GFSK & 10 \\
\hline $\begin{array}{c}\text { Traceback depth of GFSK } \\
\text { demodulator }\end{array}$ & 45 \\
\hline Distance [cm] & \\
\hline
\end{tabular}

The performances are evaluated using a bit error rate (BER) as well as frame error rate (FER). BER represents the number of bit errors divided by the total number of transferred bits. The FER is the number of corrupted frames divided by the total number of transmitted frames. 


\section{B. Results in AWGN channel}

A first approach to evaluate the performances of BLE and SmartBAN has been made only in a AWGN channel to see how the BER changes with different values of energy per bit to the spectral noise density ratio $\left(E_{\mathrm{b}} / N_{0}\right)$. The results comparing the BERs of these two technologies are shown in Figure 6.

In Figure 6, the SmartBAN curve is drawn by using the PPDU repetition of 1 , being the worst BER curve from an analysis, as seen in a previous analysis on SmartBAN in [10].

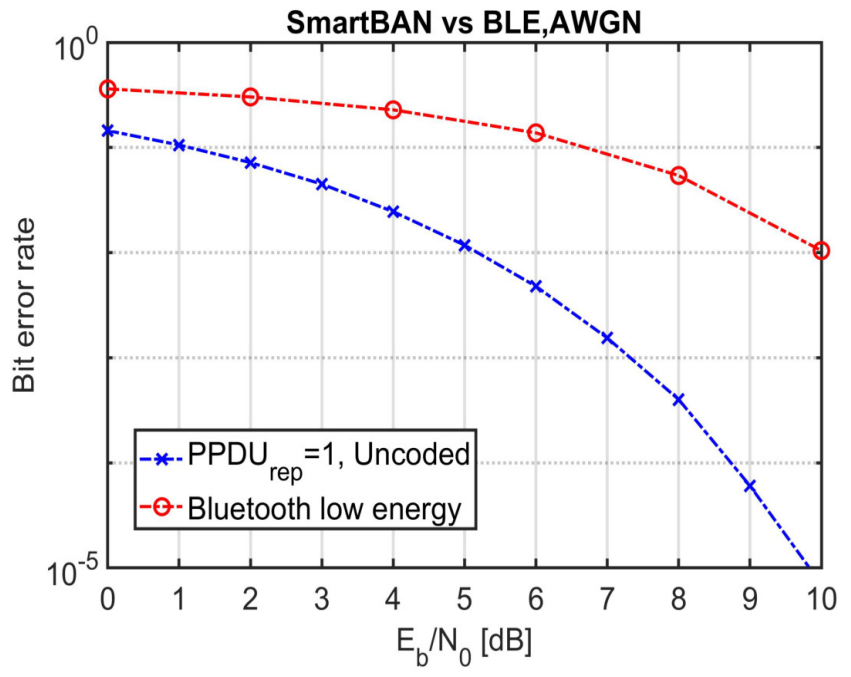

Figure 6. BER performance in the AWGN for SmartBAN and BLE.

The SmartBAN always show a better performance in terms of BER. BLE starts with 0.364 and the SmartBAN at 0.18 at $E_{\mathrm{b}} / N_{0}=0 \mathrm{~dB}$. Increasing $E_{\mathrm{b}} / N_{0}$, the performance of the SmartBAN increases drastically reaching a BER of $10^{-5}$ with a $E_{\mathrm{b}} / N_{0}=7.5 \mathrm{~dB}$, BLE requires much higher $E_{\mathrm{b}} / N_{0}$ for the same performance.

\section{Results in fading channel}

The channel model is described in the IEEE 802.15.6 CM3. For the SmartBAN system, different MAC frame sizes have been used: 50, 250, 500 and 1000 octets with a PPDU repetition of 1 since it represents the worst-case scenario for SmartBAN [10]. For the BLE, BER and FER needed for the comparison are derived. The MAC layer is not going to affect the performance in the PHY comparison between these two technologies and it is not modeled into the simulator.

In Figure 7, a performance comparison between SmartBAN and BLE is given and shows the BER performance comparison between SmartBAN and BLE: even with fading channel the SmartBAN works better than the BLE, even with the lowest MAC frame size. Anyway, it can be highlighted the difference of performance is less accentuated than the previous case with the presence of only AWGN.

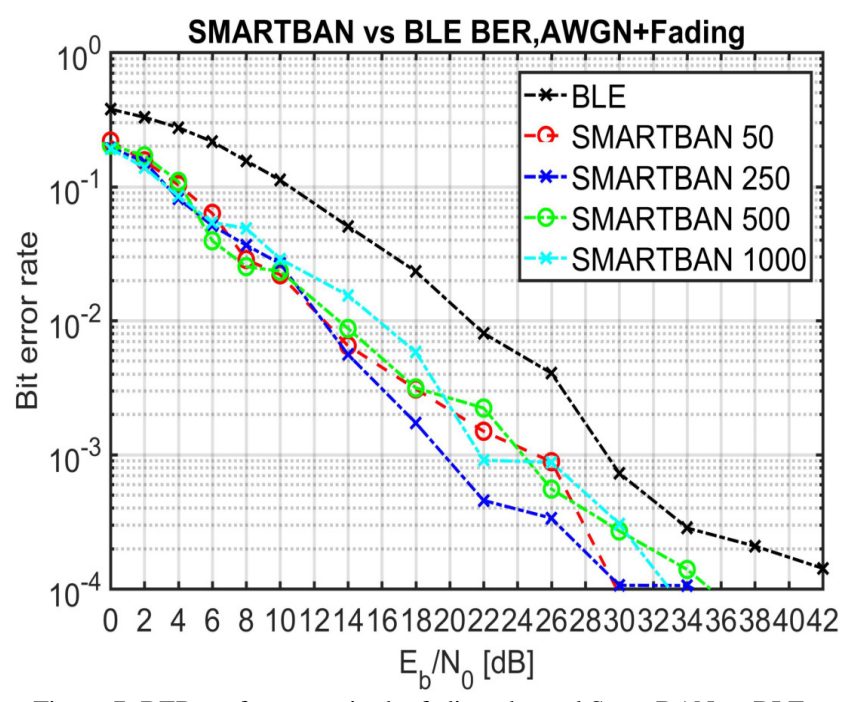

Figure 7. BER performance in the fading channel SmartBAN vs BLE.

In Figure 8, the FER comparison between BLE and SmartBAN in fading channel is given. In this case, the performance of the two standards is almost the same and from this point of view, the two technologies can be considered to work similarly. Neither the repetition nor the encoding has been chosen for the SmartBAN to make a fair comparison with BLE, in this case. Adding repetition and encoding to SmartBAN surely lead to a FER curve below the BLE one.

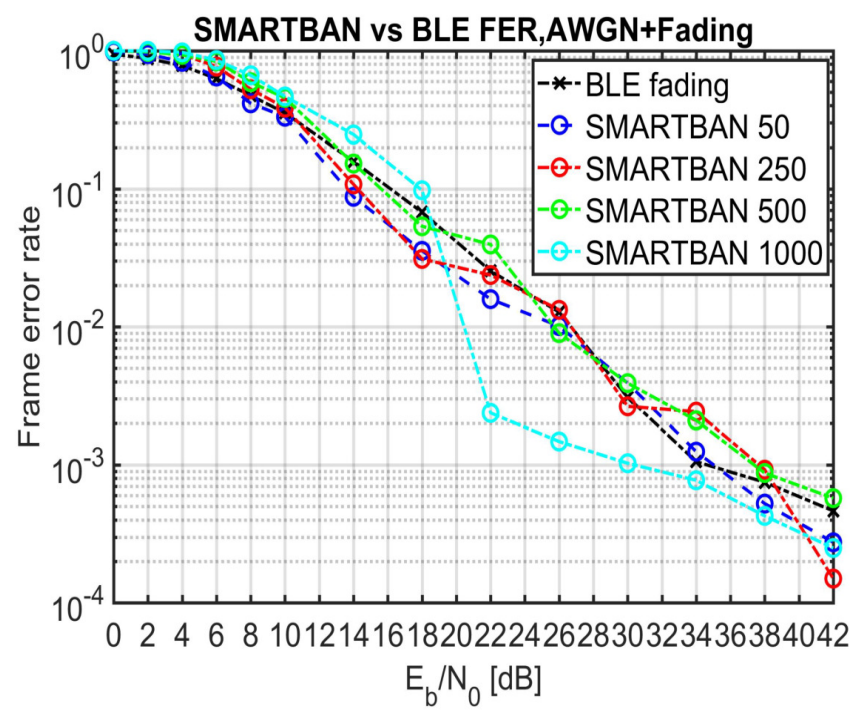

Figure 8. FER performance in the fading channel SmartBAN vs BLE.

\section{CONCLUSIONS}

This paper introduced the simulation results of the performance of SmartBAN technology compared with the BLE technology.

During the studies, a simulation environment using MATLAB software has been developed. By analyzing BER and FER results, it is possible to determine the performance of SmartBAN and BLE. For SmartBAN, the worst case 
scenario selected from the previously published results has been chosen. For BLE, the standard does not present encoding and repetition, so for a fair comparison, also SmartBAN performance was analyzed without. Concerning the BER performance, with no doubt, the SmartBAN standard for healthcare applications performs better than BLE. In terms of FER, the two technologies showed similar performance.

Future work will include a comparison with the new technology of BLE version 5.0, which presents encoding and a comparison between the technologies considering also the interference models used in the SmartBAN standard.

\section{ACKNOWLEDGMENT}

Authors would like to thank members of ETSI TC SmartBAN for fruitful discussions and co-operation.

\section{REFERENCES}

[1] http://www.etsi.org/technologies-clusters/technologies/smart-bodyarea-networks

[2] ETSI, Smart Body Area Network (SmartBAN); Enhanced Ultra-Low Power Physical Layer. ETSI TS 103326.

[3] ETSI, Smart Body Area Network (SmartBAN); Low Complexity Medium Access Control (MAC) for SmartBAN. ETSI TS 103325.

[4] ETSI, Smart Body Area Networks (SmartBAN) Unified data representation formats, semantic and open data model. ETSI TS 103378.

[5] ETSI, Smart Body Area Network (SmartBan); Measurements and modelling of SmartBAN Radio Frequency (RF) environment. ETSI TR 103395 .

[6] M. Hämäläinen, T. Paso, L. Mucchi, M. Girod-Genet, J. Farserotu, H. Tanaka, W.H. Chin, L. Nachabe, "ETSI TC SmartBAN: Overview of the Wireless Body Area Network Standard", The 9th International Symposium on Medical Information and Communication Technology (ISMICT2015), 24-26.3.2015, Kamakura, Japan.

[7] W.H. Chin, H. Tanaka, T. Nakanishi, T. Paso, M. Hämäläinen, "An Overview of ETSI TC SmartBAN's Ultra Lower Power Physical Layer", The 9th International Symposium on Medical Information and Communication Technology (ISMICT2015), 24-26.3.2015, Kamakura, Japan.
[8] K. Y. Yazdandoost, K. Sayrafian-Pour, "Channel model for body area network (BAN)", Apr. 2009.

[9] M.H. Virk, R. Vuohtoniemi, M. Hämäläinen, J. Iinatti, J-P. Mäkelä, "Stochastic Spectral Occupancy Modeling: A Body Area Network Perspective in ISM Band", The 9th International Symposium on Medical Information and Communication Technology (ISMICT2015), 24-26.3.2015, Kamakura, Japan.

[10] H. Viittala, L. Mucchi, M. Hämäläinen, T. Paso: "ETSI SmartBAN System Performance and Coexistence Verification for Healthcare", IEEE Access, Dec. 2017, Volume: 5, Issue: 1, pp: 8175-8182. DOI: 10.1109/ACCESS.2017.2697502.

[11] H. Viittala, L. Mucchi, M. Hämäläinen, "Performance of the ETSI SmartBAN System in the Interfered IEEE 802.15.6 Channel", the 11th International Symposium on Medical Information and Communication Technology (ISMICT2017), 6-8.2.2017, Lisbon, Portugal.

[12] Bluetooth Specification version 4.2, The Bluetooth Special Interest Group,Kirkland, WA, USA, 2013. P 2565

[13] K. Mikhaylov et.al., "Performance analysis and comparison of Bluetooth Low Energy with IEEE 802.15.4 and SimpliciTI," J. Sens. Actuator Networks, vol. 2, no. 3, pp. 589-613, Aug. 2013.

[14] Mikhaylov, K., \& Tervonen, J. (2013, November). Multihop data transfer service for Bluetooth Low Energy. In ITS Telecommunications (ITST), 2013 13th International Conference on (pp. 319-324). IEEE.

[15] BLE CHANNLES FIGURE http://www.rfwirelessworld.com/Tutorials/Bluetooth-Smart-Bluetooth-Low-Energy-BLEtutorial.html

[16] Jia Liu, Canfeng Chen, and Yan Ma, "Modeling Neighbor Discovery in Bluetooth Low Energy Networks"

[17] Carles Gomez,1, Joaquim Oller, and Josep Paradells, "Overview and Evaluation of Bluetooth Low Energy: An Emerging Low-Power Wireless Technology"

[18] K.Y. Yazdandoost and K. Sayrafian-Pour, "Channel Model for BodyArea Network (BAN)," IEEE P802.15 Wireless Personal Area Networks, Tech.Rep. IEEE P802.15-08-780-09-0006, April,2009.

[19] Specialist Task Force 511: SmartBAN Performance and Coexistence Evaluation, accesed on Apr. 28, 2017. [Online]. Available: https://portal.etsi.org/STF/stfs/STFHomePages/STF511. 